\title{
Le secteur agricole dans les marchés du carbone ?
}

\author{
Alexia LESEUR \\ Mission Climat de la Caisse des Dépots, CDC, \\ 56 rue de Lille, F-75007 Paris \\ <alexia.leseur@caissedesdepots.fr>
}

\begin{abstract}
Fighting against climate change in an efficient way must involve all countries, which is partly done thanks to the Kyoto protocol. At the French level, the action should involve all economic sectors and agriculture in particular. This sector can contribute by three ways: reducing directly its emissions, substituting fossil energy by biomass, and sequestrating carbon. However, in the scheme of the common agricultural policy, there is hardly an incentive or a financial remuneration to reduce greenhouse gas emissions in France.

Financial benefit from emission reductions is yet already possible on carbon markets, at the international level via the Kyoto protocol and at the European level via the emissions trading system. After drawing up the inventory of greenhouse gas emissions from agriculture and an overview of the different carbon markets, we explain how agriculture can be involved, thanks to "domestic offset projects".
\end{abstract}

Key words: carbon markets, climate change, domestic offset projects, greenhouse gas, agriculture

projets agricoles potentiellement réducteurs d'émissions de gaz à effet de serre. Ensuite, nous présentons un panorama général des marchés actuels du carbone en détaillant la place qui est faite à l'agriculture dans ces marchés. Enfin, une proposition d'intégration du secteur de l'agriculture dans le marché du carbone est avancée, via un système de « projets domestiques».

\section{Les émissions de gaz à effet de serre en agriculture : un état des lieux}

Même si elle a déjà un rôle positif dans la lutte contre l'effet de serre via sa capacité à séquestrer du carbone dans la biomasse grâce à la photosynthèse, l'agriculture est, en France, le troisième secteur émetteur de gaz à effet de serre (GES), derrière le transport et l'industrie manufacturière, avec $107,9 \mathrm{MteqCO}_{2}$ (millions de tonnes équivalent $\mathrm{CO}_{2}$ ) émises en 2003. Cela représente environ $20 \%$ des émissions françaises de GES (hors prise en compte des " puits » permettant la séquestration du carbone).

Le secteur agricole, contrairement aux autres secteurs (transport, bâtiment, industries), se caractérise par des émissions très importantes de méthane $\left(\mathrm{CH}_{4}\right)$ et de protoxyde d'azote $\left(\mathrm{N}_{2} \mathrm{O}\right)$, deux $\mathrm{GES}$ à fort pouvoir de réchauffement, et par une faible émission de dioxyde de carbone $\left(\mathrm{CO}_{2}\right)$.

Les émissions du secteur agricole présentent la particularité d'être très dispersées, puisque ce sont les 664000 exploitations de France, toutes productions confondues, qui en sont responsables.

Les émissions du secteur agricole sont plus difficiles à calculer que celles d'autres secteurs comme l'industrie ou le bâtiment. Elles dépen- dent en effet de multiples paramètres, qui sont eux-mêmes difficilement observables (le type de sol et les conditions climatiques locales influencent beaucoup les émissions de $\mathrm{N}_{2} \mathrm{O}$ liées à la fertilisation azotée par exemple) ${ }^{1}$. Le tableau 1 récapitule les sources agricoles des émissions, leur contribution, et leur évolution depuis 1990. II est à noter que l'agriculture a réduit de façon significative ses émissions depuis cette date.

\section{Un réel potentiel de réduction des émissions de GES}

Un groupe de travail, réunissant tant des représentants d'agriculteurs que des industriels, des instituts professionnels, des experts de l'Inra et des représentants des pouvoirs publics, a été créé en janvier 2005, en partenariat avec la Société des agriculteurs de France (SAF) et la Caisse des dépôts, pour identifier les principaux leviers d'action de réduction d'émission dans le secteur agricole, reprenant et affinant ainsi d'autres études précédentes [3, 4]. Un premier résultat a été mis en évidence : le secteur agricole et forestier peut contribuer en tant que tel à la réduction des émissions de gaz à effet de serre, et il peut aussi engendrer de fortes réductions d'émissions dans les autres secteurs de l'économie française, en amont et en aval.

\footnotetext{
${ }^{1}$ Dans l'inventaire national répertoriant l'ensemble des émissions françaises de GES, le CITEPA (Centre interprofessionnel technique d'études de la pollution atmosphérique) a dû retenir un certain nombre de règles comptables issues des recommandations des scientifiques du GIEC (Groupe intergouvernemental d'experts sur l'évolution du climat).
} 
Tableau 1. La contribution des différentes sources agricoles d'émission et son évolution sur la période 1990-2003 (source: CITEPA, 2004, format PNLCC) [2].

\begin{tabular}{|c|c|c|c|}
\hline Source & Gaz concernés & $\begin{array}{l}\text { Émissions totales } \\
\text { en } 2003\left(\mathrm{Mteq} \mathrm{CO}_{2}\right)\end{array}$ & $\begin{array}{c}\text { Évolution } \\
\text { des émissions } \\
(1990-2003)\end{array}$ \\
\hline Consommation d'énergie & $\mathrm{CO}_{2}$ & 10,0 & $-7 \%$ \\
\hline Cultures et pâturages & $\mathrm{N}_{2} \mathrm{O}$ & 50,1 & $-11 \%$ \\
\hline Fermentation entérique & $\mathrm{CH}_{4}$ & 28,3 & $-8 \%$ \\
\hline Déjections animales & $\mathrm{CH}_{4}$ et $\mathrm{N}_{2} \mathrm{O}$ & 19,4 & $-6 \%$ \\
\hline Riziculture & $\mathrm{CH}_{4}$ & 0,1 & $0 \%$ \\
\hline TOTAL & & 107,9 & $-9 \%$ \\
\hline
\end{tabular}

\section{Exemples de projets réducteurs d'émissions}

Pour chacune des sources d'émission, le groupe de travail a identifié des types de projet particulièrement intéressants :

- Changement d'une chaudière fuel ou gaz pour une chaudière paille ou bois, réduction de la consommation énergétique sur l'exploitation (tracteurs, énergie consommée par les bâtiments, serres, et autres chaufferies) : pour toute tonne de fuel économisée, on évite le rejet dans l'atmosphère d'environ 3 teq $\mathrm{CO}_{2}$ [5].

- Développer les biocarburants, qui se substituent à de l'énergie fossile. Chaque tonne de biocarburant permettrait d'économiser environ 2,3 teq $\mathrm{CO}_{2}$ par rapport à son équivalent fossile, sur l'ensemble du cycle de vie [6].

- Récupérer le méthane des déjections porcines ou bovines et le valoriser énergétiquement : sur un élevage de 200 truies mères, la méthanisation permettrait d'éviter environ 220 teq $\mathrm{CO}_{2}$ /an.

- Maîtriser la fertilisation : 1 tonne d'engrais azotée évitée correspond à une réduction d'émission de 10 teq $\mathrm{CO}_{2}$.

\section{Trois familles de projets}

Ces différents types de projet peuvent se regrouper en trois familles:

- Certains projets reposent sur des technologies éprouvées et sont relativement simples à mettre en œuvre. C'est notamment le cas des projets visant à utiliser la biomasse (paille, bois, cultures dédiées) à des fins énergétiques, des projets d'optimisation des transports d'intrants ou de produits agricoles, et des projets de méthanisation des déjections. Ces projets sont d'ailleurs déjà mis en œuvre avec succès, à l'étranger, dans le cadre des mécanismes de projets prévus par le protocole de Kyoto (cf. section suivante).

- D'autres projets prometteurs reposent sur des technologies ou des méthodes de mesure des émissions évitées qui demandent une expertise scientifique ou technique complémentaire. ॥ s'agit notamment des projets liés à l'alimentation des bovins, ou à la maîtrise de la fertilisation sur grandes cultures, ou sur prairies.

- La séquestration de $\mathrm{CO}_{2}$ par des activités agricoles ou forestières présente un potentiel important à long terme. Ces projets ne peuvent ces usines. pas être valorisés dans le cadre actuel du fait des règles comptables retenues, mais pourraient l'être après 2012. Compte tenu de ces difficultés, nous ne détaillerons pas les projets de séquestration dans cet article.

Ainsi, un certain nombre de projets réducteurs d'émission de gaz à effet de serre pourrait être réalisé, représentant un fort potentiel. Le tableau 2 résume le potentiel maximal de réduction de gaz à effet de serre pouvant être obtenu via ces projets. II s'agit d'un potentiel maximal, tenant compte de la faisabilité technique des projets. Pour estimer précisément les réductions prévisibles, il conviendrait d'engager des études technico-économiques fines pour évaluer la rentabilité précise du projet et des études socio-environnementales mettant I'accent sur les impacts globaux du projet, notamment hors GES.

Pour certains types de projet, il existe déjà une politique publique qui les favorise : c'est le cas

Tableau 2. Le potentiel de réduction des émissions de GES en agriculture [7].

\begin{tabular}{|c|c|c|}
\hline Sous-secteur & Levier d'action & $\begin{array}{c}\text { Potentiel } \\
\text { (tonnes équivalent } \mathrm{CO}_{2} / \mathrm{an} \text { ) }\end{array}$ \\
\hline \multicolumn{3}{|c|}{ Énergie : potentiel de $15 \mathrm{MteqCO}_{2}$ par an (hors biocarburants et biomatériaux) } \\
\hline \multirow[t]{5}{*}{$\begin{array}{l}\text { Énergie : substitution à } \\
\text { l'énergie fossile }\end{array}$} & $\begin{array}{l}\text { Réduction de consommation d'énergie } \\
\text { sur l'exploitation, par des itinéraires } \\
\text { techniques simplifiés }\end{array}$ & 400000 \\
\hline & Chaudière paille-énergie & 2400000 \\
\hline & Chaudière cultures dédiées & 5850000 \\
\hline & Chaudière bois-énergie & 6300000 \\
\hline & Biocarburants $^{\mathrm{a}}$ & 7000000 \\
\hline Biomatériaux & Bois-matériau - émissions évitées ${ }^{b}$ & 900000 \\
\hline \multicolumn{3}{|c|}{ Élevage : potentiel de $5,9 \mathrm{Mteq} \mathrm{CO}_{2}$ par an } \\
\hline \multirow[t]{2}{*}{ Gestion des déjections } & $\begin{array}{l}\text { Méthanisation - émissions évitées de } \\
\mathrm{CH}_{4} \text { et de } \mathrm{N}_{2} \mathrm{O}\end{array}$ & 2200000 \\
\hline & Méthanisation - substitution d'énergie & 1200000 \\
\hline \multirow[t]{3}{*}{ Alimentation } & $\begin{array}{l}\text { Alimentation des bovins - diminution } \\
\text { de la fermentation entérique }\end{array}$ & 475000 \\
\hline & $\begin{array}{l}\text { Alimentation des bovins - diminution } \\
\text { des déjections }\end{array}$ & 235000 \\
\hline & $\begin{array}{l}\text { Augmentation de la productivité des } \\
\text { bovins }\end{array}$ & 770000 \\
\hline Gestion des prairies & Semis de trèfles en prairies & 1000000 \\
\hline \multicolumn{3}{|c|}{ Grandes cultures : potentiel de $1,2 \mathrm{MteqCO}_{2}$ par an } \\
\hline \multirow[t]{2}{*}{ Grandes cultures } & Fertilisation maîtrisée & 900000 \\
\hline & $\begin{array}{l}\text { Utilisation d'engrais verts en } \\
\text { interculture }\end{array}$ & 350000 \\
\hline
\end{tabular}

a L'objectif annoncé d'incorporation de 5,75\% de biocarburants dans les carburants d'origine fossile d'ici 2010 représente une réduction des émissions de 7000000 teq $\mathrm{CO}_{2} /$ an, d'après le Plan Climat [3]. Du fait de cet objectif et des mesures correspondantes en cours de mise en place, il semble difficile de considérer que des projets domestiques pourraient concerner le développement des biocarburants, sauf si leur additionnalité par rapport à cet objectif est démontrée. Par exemple, il serait également possible de réduire les émissions liées à la production de biocarburants, notamment les émissions de $\mathrm{N}_{2} \mathrm{O}$ lors de la phase culturale du colza, et les émissions de $\mathrm{CO}_{2}$ lors de la phase industrielle pour la première transformation du blé et de la betterave.

${ }^{b}$ Le bois matériau se substitue avec des matériaux dont la production est très énergivore comme le ciment ou l'acier, mais la valorisation des réductions d'émissions est déjà permise, via le système de quotas qui couvrent 
notamment des biocarburants, pour lesquels il a été décidé un objectif $d$ 'incorporation de $5,75 \%$ dans les carburants d'origine fossile d'ici 2010. Mais excepté ce cas, la politique agricole actuelle $n^{\prime}$ incite pas suffisamment ces bonnes pratiques: la fiscalité, la réglementation, et les écoconditionnalités qui sont désormais associées aux aides à l'agriculture, ou les aides du deuxième pilier de la PAC tiennent encore peu compte des émissions de gaz à effet de serre. Or, la fiscalité pourrait par exemple être un instrument incitatif efficace pour réduire les émissions de gaz à effet de serre liées à l'épandage d'engrais azotés; de même, la conditionnalité des aides pourrait englober par exemple un volet «effet de serre ». Mais l'incorporation de cet aspect n'a pas fait l'objet d'une étude approfondie dans le cadre de ce travail, qui a été plutôt axé sur les marchés du carbone et la valorisation possible de ces projets dans ce cadre. Ainsi, le tableau 2 mentionne mais ne comptabilise pas les projets qui ne pourront pas être éligibles aux «projets domestiques » comme ceux liés aux biocarburants et au bois-matériau (cf. section suivante).

\section{La valorisation économique de ces réductions dans le domaine agricole : via les marchés du carbone?}

La valorisation financière des réductions d'émissions est déjà possible, dans certains cas, sur les différents marchés du carbone. Mais le secteur agricole est peu encore présent. Détaillons ces marchés et la place qu'ils réservent à l'agriculture.

\section{Les différents marchés du carbone existants et la place de l'agriculture}

Il existe, non pas un mais plusieurs marchés du carbone, et plusieurs monnaies associées: la monnaie "État », la monnaie " projet », et une autre indépendante du protocole de Kyoto qui est le quota européen.

\section{Le marché international des UQA, et les projets MOC et MDP}

Face au problème du changement climatique, les pays développés se sont individuellement engagés, en 1997, dans le protocole de Kyoto, à réduire leurs émissions de gaz à effet de serre pour la période 2008-2012 par rapport à leurs émissions de 1990. Ces engagements portent sur l'ensemble des 6 gaz à effet de serre d'origine humaine $\left(\mathrm{CO}_{2}, \mathrm{CH}_{4}, \mathrm{~N}_{2} \mathrm{O}, \mathrm{HFC}, \mathrm{PFC}, \mathrm{SF}_{6}\right)$. Pour satisfaire cet objectif, chaque pays signataire peut mettre en place des politiques et mesures lui permettant de réduire les émissions sur son sol national. Le protocole de Kyoto I'autorise également à prendre part à un marché d'UQA (unités de quantités attribuées) lui permettant d'acheter des réductions d'émissions faites dans d'autres pays signataires $d u$ protocole.

Ainsi, I'objectif environnemental est atteint, et l'est à un moindre coût : grâce à ce marché, les pays ayant pris des engagements peuvent atteindre leur objectif de réduction à un coût plus faible qu'en l'absence de ce marché, puisqu'ils ne sont pas alors obligés d'engager sur leur territoire des réductions d'émissions très coûteuses qui pourraient nuire aux différents secteurs d'activités. Les réductions d'émissions seront faites là où elles sont les moins onéreuses, ce qui permet de ne pas mettre en péril le développement économique des pays.

Le protocole de Kyoto permet également le recours à deux autres mécanismes par projets, qui sont déjà effectifs, et qui s'appuient sur des projets réducteurs d'émissions mis en œuvre dans différents pays :

\section{Le mécanisme pour un développement propre} (MDP)

II se traduit par un investissement d'un pays développé dans un PVD pour un projet permettant de réduire les émissions de GES par rapport à un scénario tendanciel. Cet effort est valorisé, après vérification de la réalité des réductions d'émissions, par l'octroi de crédits d'émission, appelés URCE (unités de réduction certifiée d'émissions). L'investisseur se rémunère ainsi en partie en crédits d'émission, qu'il peut revendre sur le marché international. On comptait ainsi, en 2004, 107 Mteq $\mathrm{CO}_{2}$ échangés dans le monde, issus de mécanismes de projets, pour un prix entre 3 et $8 \$[8]$.

\section{Le mécanisme de mise en œuvre conjointe (MOC)}

Il concerne les investissements effectués par un pays développé dans un autre pays développé ayant contracté un engagement de réduction d'émissions dans le cadre du protocole de Kyoto. Cet effort est valorisé par la conversion, par le pays hôte, d'une partie de ses unités de quantité attribuées (UQA) dans le cadre du protocole de Kyoto en crédits d'émission, appelés URE (unités de réduction d'émissions). Là encore, l'investisseur se rémunère en partie en crédits d'émission, négociables sur le marché. Les secteurs agricole et forestier participent déjà à la réduction d'émission de GES, dans le cadre du protocole de Kyoto: en effet, de nombreux projets MDP et MOC concernent ces secteurs. Au total, plus de cinquante projets étaient en cours de validation fin juin 2005. La carte de la figure 1 fournit pour les principaux pays promoteurs les types de projets agricoles les plus fréquemment mis en œuvre. La plupart des projets concernent des réductions d'émissions soit de $\mathrm{CO}_{2}$ par production de chaleur ou d'électricité à partir de biomasse (en bleu), soit de $\mathrm{CH}_{4}$ ou de $\mathrm{N}_{2} \mathrm{O}$ liées à la gestion des effluents d'élevage (en rouge) qui est essentiellement du traitement du lisier

\section{Le marché européen des quotas}

Depuis le début de l'année 2005 s'est mis en place un marché européen de quotas ${ }^{2}$ d'émission de $\mathrm{CO}_{2}$. Un nouveau monde vient donc de s'ouvrir en Europe : celui où l'on pénalise les acteurs qui accroissent leurs émissions et où l'on rémunère ceux qui les réduisent. Mais, pour l'instant, cette valorisation financière des réductions d'émissions ne concerne que certains acteurs économiques, à savoir les industries fortement émettrices de $\mathrm{CO}_{2}$ : installations de combustion de plus de $20 \mathrm{MW}$, énergéticiens, sidérurgie et industrie papetière. Au total, seules $30 \%$ des émissions françaises de gaz à effet de serre sont touchées par ce mécanisme financier et le secteur agricole $n^{\prime}$ est pas directement concerné. Le caractère dispersé des émissions agricoles fait qu'elle ne sera vraisemblablement jamais concernée par un tel système : en effet, il serait très lourd d'un point de vue administratif, et très coûteux, d'intégrer les 660000 exploitations françaises dans le système des quotas européen, lesquelles individuellement émettent peu de gaz à effet de serre.

On peut en revanche signaler deux faits importants concernant le secteur agricole et forestier :

- d'une part, même si le secteur agricole n'est pas couvert par les quotas de $\mathrm{CO}_{2}$, les secteurs amont et aval de l'agriculture le sont. En effet, sur les 156 millions de quotas annuels du PNAQ français, 7,2 millions sont ainsi alloués aux 158 industries agroalimentaires comportant des installations de combustion de plus de $20 \mathrm{MW}$ : industries de l'amidon, brasserie, distilleries, usines de production d'huile, laiteries, sucreries, etc. Par ailleurs, certaines industries de production d'intrants, en particulier d'engrais, sont concernées par les quotas en raison de leurs émissions lors du processus industriel. Enfin, certaines industries et certaines collectivités locales possédant des installations d'une puissance supérieure à $20 \mathrm{MW}$ sont également concernées par la directive quotas : si ces industries ou ces collectivités changent leurs installations pour utiliser de la biomasse comme combustible (bois, paille, etc.), les

2 Pour un panorama général du marché européen des quotas, cf. Mission Climat, 2006 [9]. 


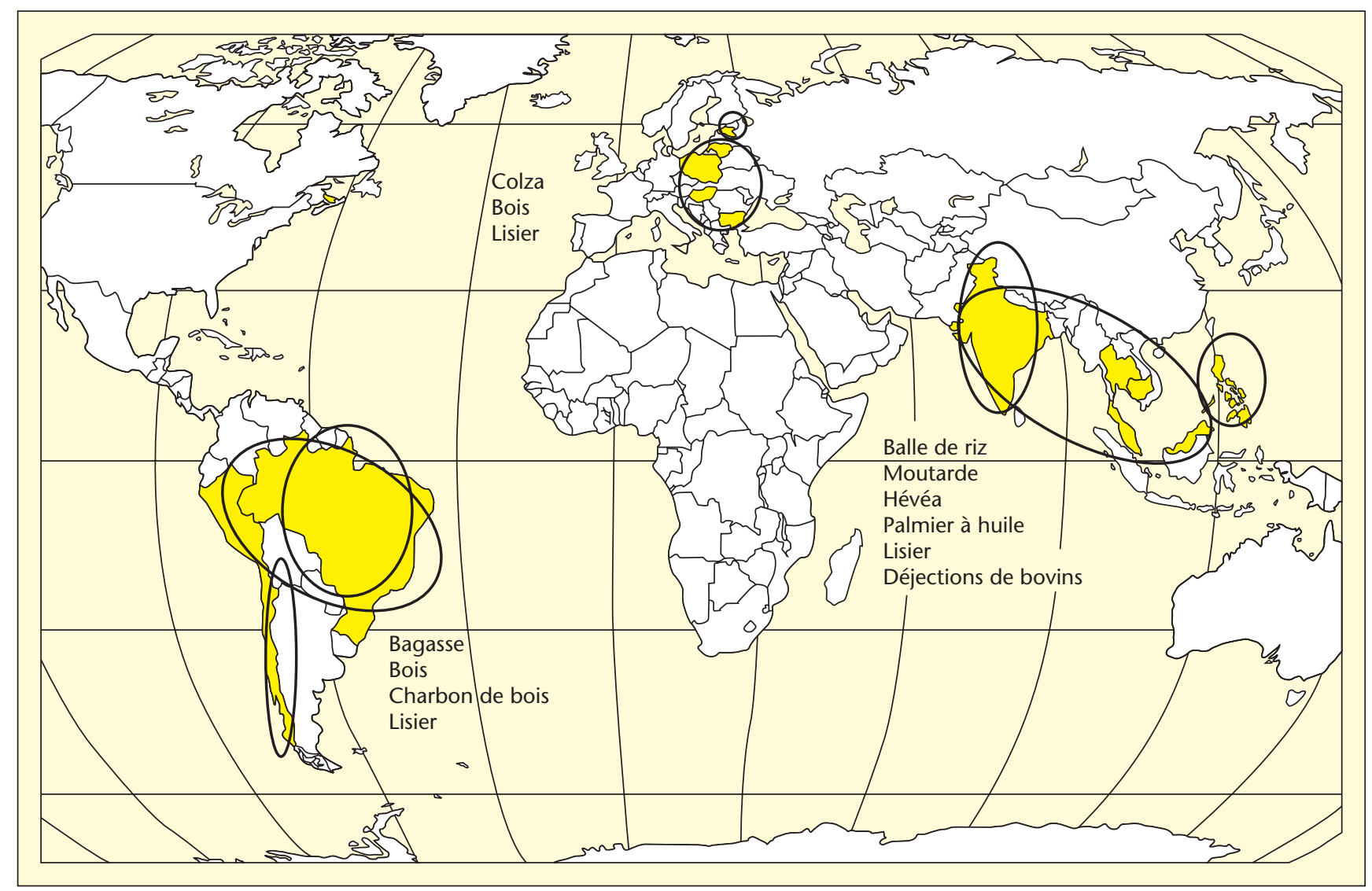

Figure 1. Les projets agricoles MOC et MDP dans le monde [7].

réductions d'émission ${ }^{3}$ ainsi permises seront valorisées via le système des quotas ;

- d'autre part, puisque les quotas et les crédits carbone URCE et URE issus de mécanismes de projets (MDP et MOC) sont fongibles (au sens où l'un peut tenir lieu de l'autre), un industriel agroalimentaire français ayant par exemple des activités agricoles en Allemagne et au Maroc peut, si ces pays l'acceptent, valoriser des réductions d'émissions liées à des projets agricoles dans le cadre de la MOC en Allemagne ou du MDP au Maroc, et vendre ces crédits sur le marché européen des quotas. En revanche, il ne pourra espérer, dans le cadre juridique actuel, valoriser les réductions d'émissions du même type de projet en France.

\footnotetext{
${ }^{3}$ Dans l'inventaire national, la combustion de la biomasse n'est pas comptabilisée comme une émission, puisqu'il s'agit d'un retour à l'atmosphère du carbone qui a été séquestré dans les végétaux. De ce point de vue, les émissions comptabilisées sont nulles; en revanche, il peut exister des fuites, dues aux émissions liées au transport de la biomasse, notamment de la paille puisque celle-ci est relativement peu dense.
}

\section{L'intérêt des projets domestiques}

Deux conclusions peuvent être tirées du panorama des différents marchés du carbone et de la place que chacun d'eux réserve à l'agriculture: d'une part, le principe du marché du carbone permet d'envoyer un signal-prix juste et efficace quant aux mesures de réductions d'émissions qu'il est pertinent de mettre en œuvre et de rémunérer les porteurs de projets ; d'autre part, l'agriculture est peu concernée par ces marchés et uniquement au niveau international. Le protocole de Kyoto permet en effet à un investisseur français d'investir à l'étranger dans des projets agricoles réducteurs d'émissions et de valoriser les émissions évitées. En revanche, le cadre actuel ne lui permet pas d'être rémunéré pour la mise en œuvre de tels projets réducteurs d'émissions dans l'agriculture française.

Pour répondre à cette insuffisance et permettre à l'agriculture de prendre part à la lutte contre l'effet de serre et en tirer certains bénéfices, nous avons développé l'idée de "projet domestique » [10]. À l'image de certains marchés du carbone existants dans le monde qui intègrent le secteur agricole (les projets MDP et
$\mathrm{MOC}$ agricoles, et les projets domestiques mis en œuvre dans des pays comme le Canada, la Nouvelle-Zélande, les États-Unis et l'Australie), un système de projets domestiques pourrait être expérimenté en France et permettre une rémunération des porteurs de projets dans le secteur agricole, et ce, sans subvention de la part de l'État ou de l'Union européenne. Le principe d'un projet domestique est de permettre une valorisation financière des réductions d'émissions de gaz à effet de serre, en I'occurrence dans les secteurs agricole et forestier, via l'attribution de crédits carbone ou de leur valeur correspondante en fonction des réductions d'émission permises. En ce sens, il est similaire aux mécanismes de projets du protocole de Kyoto, et en particulier à la MOC, puisqu'il est assis sur une conversion des actifs UQA du pays concerné en actifs utilisables pour les agents.

Un système de projet domestique consisterait en trois phases:

- La mise en place du projet et une évaluation des émissions évitées par rapport aux émissions de GES qui auraient eu lieu en l'absence du projet. 
- La vérification que ce projet répond à des critères de développement durable.

- Une rémunération financière octroyée au porteur de projet en fonction de la quantité d'émissions évitées.

Pour la bonne mise en œuvre du système, ces projets devront être promus et développés par des porteurs de projets, qui agiront comme des points de regroupement auprès d'un nombre suffisant d'exploitations agricoles. Les projets portés par l'ensemble d'une filière sont donc particulièrement visés et prometteurs, d'autant plus qu'ils pourraient comporter plusieurs volets liés à la réduction des émissions (diminution de la fertilisation et optimisation des transports par exemple). Les porteurs de projets pourraient être, suivant les cas, des coopératives agricoles, des associations d'exploitants ou encore des industriels.

La rémunération du porteur de projet pourrait prendre différentes formes: I'attribution de " crédits carbone » négociables sur le marché, ou plus simplement une enveloppe budgétaire ou un système de prêts bonifiés. Ces projets domestiques pourraient également bénéficier financièrement aux exploitants agricoles, en leur permettant d'une part d'avoir un nouveau débouché garanti et à long terme pour leur production et, d'autre part, de négocier leur contrat d'approvisionnement avec le porteur de projet, en tenant compte de cette valorisation des réductions d'émissions.

Ces projets ne pourront voir le jour sans l'implication des autres acteurs du monde agricole, notamment les instituts techniques, les chambres d'agriculture et les centres de gestion, et les organismes habilités pour vérifier la réduction d'émissions et sa cohérence avec l'inventaire national des émissions de GES.

\section{Conclusion}

Une lutte efficace contre l'effet de serre doit concerner l'ensemble des secteurs d'activité, parmi lesquels l'agriculture. Celle-ci présente $d^{\prime}$ ailleurs un fort potentiel de réduction des émissions de gaz à effet de serre, évalué à plus d'une quinzaine de milliers de tonnes de $\mathrm{CO}_{2}$ évitées par an, mais le cadre actuel ne lui permet pas d'être rémunéré pour la mise en œuvre de tels projets réducteurs d'émissions, alors qu'il le permet pour d'autres secteurs (les secteurs soumis au système de quotas) ou dans d'autres pays.

Du fait du caractère dispersé de ses émissions, l'agriculture ne pourra pas être intégrée au système européen d'échange de quotas. En revanche, elle peut participer au marché du carbone par d'autres voies, parmi lesquelles les projets domestiques. Ceux-ci constituent une démarche volontaire et proactive de la part du monde agricole pour se préparer à l'effort général pour "décarboner » l'économie en l'incitant à se mobiliser pour prendre sa part des efforts et des bénéfices liés à la lutte contre I'effet de serre. Des projets domestiques agricoles sont déjà mis en œuvre dans d'autres pays, notamment au Canada, aux États-Unis, en Nouvelle-Zélande et en Australie. À quand ce système en France?

\section{RÉFÉRENCES}

1. GIEC. Bilan 2001 des changements climatiques : les éléments scientifiques. IPCC. 2001. Disponible sur le site: http://www.ipcc.ch/ pub/un/giecgt1.pdf.

2. CITEPA. Inventaire des émissions de gaz à effet de serre en France au titre de la Convention cadre des Nations Unies sur les Changements Climatiques, Format CCNUCC, en 2003. 2004.
3. Ministère de l'environnement et du développement durable. Plan Climat. 2004

4. SOLAGRO. Plan Climat 2003 - 12 propositions pour lutter contre le changement climatique dans le secteur de l'agriculture. 2003. Etude pour I'ADEME et la MIES, disponible sur: http ://www.solagro.org/site/124.html.

5. WORLD RESOURCES INSTITUTE. The Greenhouse Gas Protocol : A corporate accounting and reporting standard (Revised edition), Washington DC. 2004.

6. ADEME-DIREME. Bilans énergétique des biocarburants en France. 2002.

7. Mission Climat de la Caisse des Dépôts. Société des Agriculteurs de France, Ademe. Les marchés du carbone : quelle place pour l'agriculture française? 2006. Rapport d'étude par A. LESEUR, disponible sur le site: http://www. caissedesdepots.fr/ FR/espace_presse/ publi cations_doc/etude_co2.pdf.

8. LECOCQF. Les marchés carbone dans le monde. Revue d'économie financière 2006 ; 83 : 13-30.

9. DE PeRThuis C, et al. Mission Climat de la Caisse des Dépôts (2006a) : "Marché européen : an $1^{\prime \prime}$, Lettre trimestrielle ${ }^{\circ} 5$ de la Mission Climat de laCDC, disponiblesurlesite : http ://www.caisse desdepots.fr/FR/espace presse/publications doc/lettre_mission_climat_n5.pdf.

10. Mission Climat de la Caisse des Dépôts. (2005c) : Elargir les instruments d'action contre le changement climatique grâce aux projets domestiques: Rapport d'évaluation, par DE PERTHUIS C. et al., disponible sur le site: http ://www.caissedesdepots.fr/FR/espace_ presse/publications_doc/rapport_final_projets domestiques_CO2_11_05.pdf. 